\title{
Portal Systemic Encephalopathy Presenting with Dressing and Constructional Apraxia
}

\author{
Takeshi Kanda, Shigeru Nogawa, Kazuhiro Muramatsu, Atsuo Koto and Yasuo Fukuuchi
}

\begin{abstract}
We report a case with portal systemic encephalopathy who presented with dressing and constructional apraxia and subtle weakness of the left hand. We initially suspected a cerebrovascular attack in the right cerebral hemisphere, but brain $T_{1}$-weighted magnetic resonance (MR) imaging revealed high intensity in the basal ganglia and hyperammonemia was detected. We performed abdominal MR angiography, which visualized an intrahepatic portal systemic shunt. Cerebral blood flow, measured by xenon-enhanced computed tomography, was decreased in the bilateral, but more dominantly right-sided, parietal watershed regions. We speculate that these boundary territories might be susceptible to damage by toxic metabolites of hepatic encephalopathy.
\end{abstract}

(Internal Medicine 39: 419-423, 2000)

Key words: hepatic encephalopathy, dressing apraxia, constructional apraxia, MR angiography, local cerebral blood flow, xenon-enhanced CT

\section{Introduction}

Hepatic encephalopathy is a metabolic disorder seen in patients with hepatocellular failure or a portal systemic shunt. The disease can be characterized by generalized signs, including impaired consciousness, altered neuromuscular activity, and electroencephalographic abnormalities $(1,2)$. However, it has been suggested that some patients who do not manifest such distinct symptoms can be diagnosed only by sensitive neuropsychological examination ("subclinical hepatic encephalopathy") (3). Moreover, focal neurological signs, such as hemiplegia, which sometimes mimic those of stroke, have also been described in earlier cases (4-6). Here, we report a patient with portal systemic encephalopathy (PSE) presenting with dressing and constructional apraxia, and we discuss the mechanism of these focal signs. We emphasize the clinical usefulness of cerebral and abdominal magnetic resonance imaging (MRI) in detecting an underlying portal systemic shunt.

\section{Case Report}

This 72-year-old woman noticed difficulty using the toilet and locking the door on June 16, 1998. The next day, she could not put on her clothes or use a towel after washing her face. She was admitted to our hospital on June 18, 1998. At the age of 55, she underwent heart valve replacement surgery for severe mitral regurgitation. Since the surgery, she had atrial fibrillation treated with warfarin potassium. She had a gastric ulcer at age 69, and she had suffered from constipation for the past 20 years. She did not have a history of blood transfusion or alcoholism.

On admission, the patient was $144 \mathrm{~cm}$ tall and weighed 41.5 $\mathrm{kg}$; the blood pressure was $136 / 60 \mathrm{mmHg}$; and pulse rate was 87 and irregular. She was right-handed. Physical examination was normal except for slight hepatomegaly with tenderness. Her consciousness was clear and orientation almost normal, but she was slightly disoriented for time, mistaking the admission day for the previous day. Cranial nerves and sensory system were intact. Subtle weakness of the left upper extremity was present, and the grip power of the left hand $(6 \mathrm{~kg})$ was less than that of the right hand $(18 \mathrm{~kg})$. However, deep tendon reflexes were symmetrical, and pathological reflexes were absent. Coordination was normal, but gait was slightly unstable. Flapping tremor and other involuntary movements were $a b-$ sent. Her score on the Hasegawa's dementia scale (7) was 23/ 30 points, and the revised Wechsler adult intelligence scale (WAIS-R) revealed slight impairment in visuospatial performance tasks (FIQ 104, VIQ 109, PIQ 98; block design 8). She did not have aphasia, ideomotor, or ideational apraxia. Dyscalculia was noted, although writing, finger gnosis, and right to left orientation were preserved. She could not put on her clothes, and a constructional test to copy a drawing of a simple cube was abnormal (Fig. 1). However, unilateral spatial and topographical agnosia was not detected.

Laboratory data on admission are shown in Table 1. Blood cell counts were normal. Prolonged prothrombin and thrombin time was thought to be due to anticoagulation therapy. Of the blood chemistry, IgG, both direct and indirect bilirubin, 


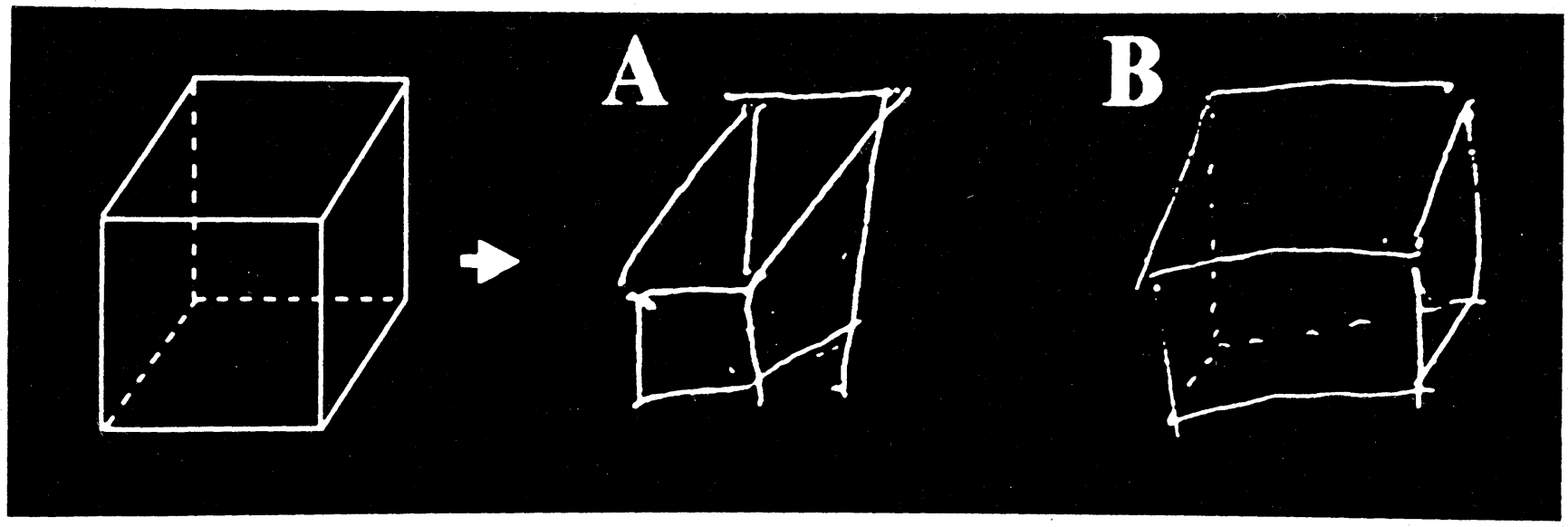

Figure 1. A: On admission, the patient could not draw the shape of a cube well. B: After treatment with low protein diet, lactulose, and branched-chain amino acids, the patient could draw it.

Table 1. Laboratory Data on Admission

\begin{tabular}{lrlr}
\hline Blood cell counts & & Lactate dehydorogenase & $282 \mathrm{IU} / l$ \\
White blood cell & $4,400 / \mu \mathrm{l}$ & Aspartate aminotransferase & $38 \mathrm{IU} / \mathrm{l}$ \\
Red blood cell & $392 \times 10^{4} / \mu l$ & Alanine aminotransferase & $26 \mathrm{IU} / l$ \\
Hemoglobin & $12.3 \mathrm{~g} / \mathrm{dl}$ & Alkaline phosphatase & $372 \mathrm{IU} / l$ \\
Hematocrit & $37.2 \%$ & $\gamma$-glutamyltranspeptide & $110 \mathrm{IU} / l$ \\
Platelet & $15.3 \times 10^{4} / \mu \mathrm{l}$ & Total cholesterol & $194 \mathrm{mg} / \mathrm{dl}$ \\
Coagulation test & & Creatine kinase & $133 \mathrm{mg} / \mathrm{dl}$ \\
Activated partial prothrombin time & $32.6 \mathrm{sec}$ & Choline esterase & $231 \mathrm{IU} / l$ \\
Prothrombin time & $37 \%$ & Tryglycerides & $43 \mathrm{~g} / \mathrm{dl}$ \\
Fibrinogen & $363 \mathrm{mg} / \mathrm{dl}$ & Amylase & $155 \mathrm{IU} / l$ \\
Thrombin time & $16 \%$ & Sodium & $136.3 \mathrm{mEq} / \mathrm{l}$ \\
TT-International Normalization Ratio & 2.11 & Potassium & $3.8 \mathrm{mEq} / l$ \\
Blood chemistry & & Chloride & $99 \mathrm{mEq} / \mathrm{l}$ \\
Total protein & $9.2 \mathrm{~g} / \mathrm{dl}$ & Calcium & $9.7 \mathrm{mg} / \mathrm{dl}$ \\
Albumin & $4.4 \mathrm{~g} / \mathrm{dl}$ & Phosphorus & $2.9 \mathrm{mg} / \mathrm{dl}$ \\
IgG & $2,410 \mathrm{mg} / \mathrm{dl}$ & Glucose & $126 \mathrm{mg} / \mathrm{dl}$ \\
IgM & $116 \mathrm{mg} / \mathrm{dl}$ & Ammonia & $82 \mathrm{mmol} / l$ \\
Total bilirubin & $3.3 \mathrm{mg} / \mathrm{dl}$ & Serological test & $0.08 \mathrm{mg} / \mathrm{dl}$ \\
Direct bilirubin & $1.2 \mathrm{mg} / \mathrm{dl}$ & C-reactive protein & $\mathrm{positive}$ \\
Urea nitrogen & $17.5 \mathrm{mg} / \mathrm{dl}$ & Antinuclear antibodies & \\
Creatinine & $0.6 \mathrm{mg} / \mathrm{dl}$ & & \\
\hline
\end{tabular}

lactate dehydrogenase, alkaline phosphatase, and $\gamma$-glutamyl transpeptidase were elevated. The anti-nuclear antibody was positive, although she did not have any signs indicating collagen diseases. The chest X-ray film showed cardiomegaly, and atrial fibrillation was present on the electrocardiogram. Mild aortic and severe tricuspid valve regurgitation with dilatation of the right atrium and the inferior vena cava was detected in the echocardiography. Blood gas analysis was normal. Brain computed tomography (CT), $\mathrm{T}_{2}$ - and diffusion-weighted images of MRI did not show any abnormality, while the $\mathrm{T}_{1}$ weighted image revealed high signal intensity in the bilateral globus pallidus (Fig. 2), suggesting chronic liver dysfunction (8-10). Then, the serum ammonia level was found to be elevated $(82 \mathrm{mmol} / \mathrm{l})$, and a low excretion of indocyanine green
15 minutes after its injection (26.4\%) was detected. EEG showed generalized high-voltage slow waves, which was compatible with hepatic encephalopathy. However, abdominal ultrasonography and liver scintigraphy showed no evidence of liver cirrhosis, and esophageal and gastric varices were not detected with upper gastrointestinal fiberscopy. These data led us to suspect portal systemic encephalopathy (PSE). We performed abdominal MR angiography, which visualized a large shunt vessel between the middle hepatic vein and the portal vein, as well as dissection of the descending aorta (Fig. 3).

To elucidate the mechanism of these focal neurological signs, we then evaluated local cerebral blood flow (LCBF) by the cold xenon-enhanced CT method. LCBF in the resting condition was decreased in bilateral, but more dominantly right-sided, 


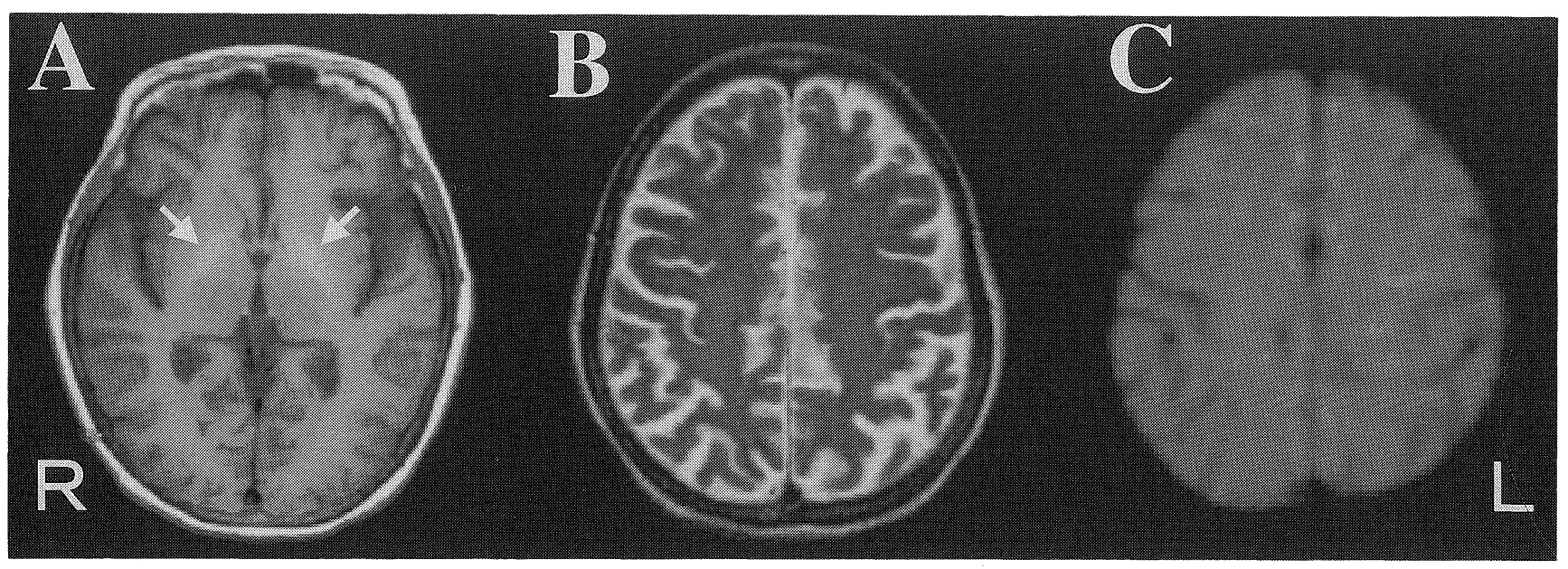

Figure 2. An axial image of $T_{1}$-weighted MRI demonstrated high signal intensity areas in the bilateral globus pallidus (arrows) consistent with hepatic encephalopathy (A). However, $\mathbf{T}_{2}$-weighted (B) or diffusion-weighted (C) MRI did not show any abnormalities in the convexity.

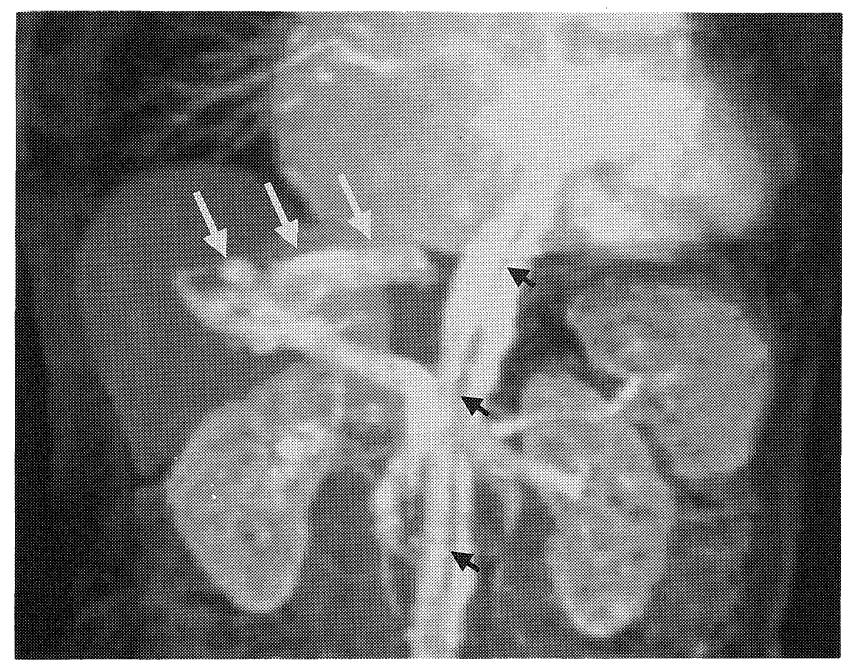

Figure 3. Coronal abdominal MR angiography showed a large shunt vessel between the middle hepatic vein and the portal vein (white arrows). A dissecting aneurysm of the descending aorta (black arrows) was also present.

parieto-occipital boundary arterial territories. The decrease in LCBF was more prominent when acetazolamide, a carbonic anhydrase inhibitor, was administered (Fig. 4). In brain MR angiography and carotid ultrasonography, however, we could not detect any stenotic lesions in the major cerebral and carotid arteries. After she was treated with a low protein diet, lactulose, and an infusion of branched-chain amino acids, the blood ammonia level was decreased. Moreover, the focal neurological signs disappeared and the constructional test was normalized (Fig. 1).

\section{Discussion}

The patient presented with dressing and constructional apraxia, which is usually caused by a parietal lesion in the nondominant hemisphere. In addition, she had subtle weakness of the left upper extremity. Because she had a prosthetic valve and atrial fibrillation, she was initially diagnosed as having a cerebrovascular attack in the right cerebral hemisphere. However, $\mathrm{T}_{2}$ - and diffusion-weighted MR images excluded an episode of acute stroke. Instead, increased $T_{1}$-signal intensity of the basal ganglia, which is closely related to PSE (8) or liver dysfunction $(9,10)$, led to the diagnosis of hepatic encephalopathy.

While fluctuation of generalized neurological signs and symptoms involving mental status, pyramidal and extrapyramidal tracts, and cerebellar systems are common in hepatic encephalopathy $(1,2)$, focal or unilateral neurological signs are usually not present (11). However, reversible focal signs, such as ocular deviation (12) or hemiplegia $(4-6,13)$, have been reported. It is conceivable that subclinical minor lesions may develop focal neurological signs when hepatic encephalopathy progresses (1). Constructional apraxia has been suggested to be a feature of mild hepatic encephalopathy (14-17). We reviewed case reports of hepatic encephalopathy and found nine cases with focal higher cortical signs $(15,17-22)$ (Table 1). Among them, constructional apraxia was observed in eight cases $(15,17,19-22)$, and dressing apraxia was found in two cases $(15,17)$.

The region responsible for dressing or constructional apraxia is the parietal cortex, which is located in the watershed area between the supply of major cerebral arteries. Because the region is hemodynamically vulnerable ("locus minoris"), neurons in the regions might be susceptible to damage by toxic metabolites or vasoparalytic substances of hepatic encephalopathy. Indeed, according to Finlayson and Superville (23), 


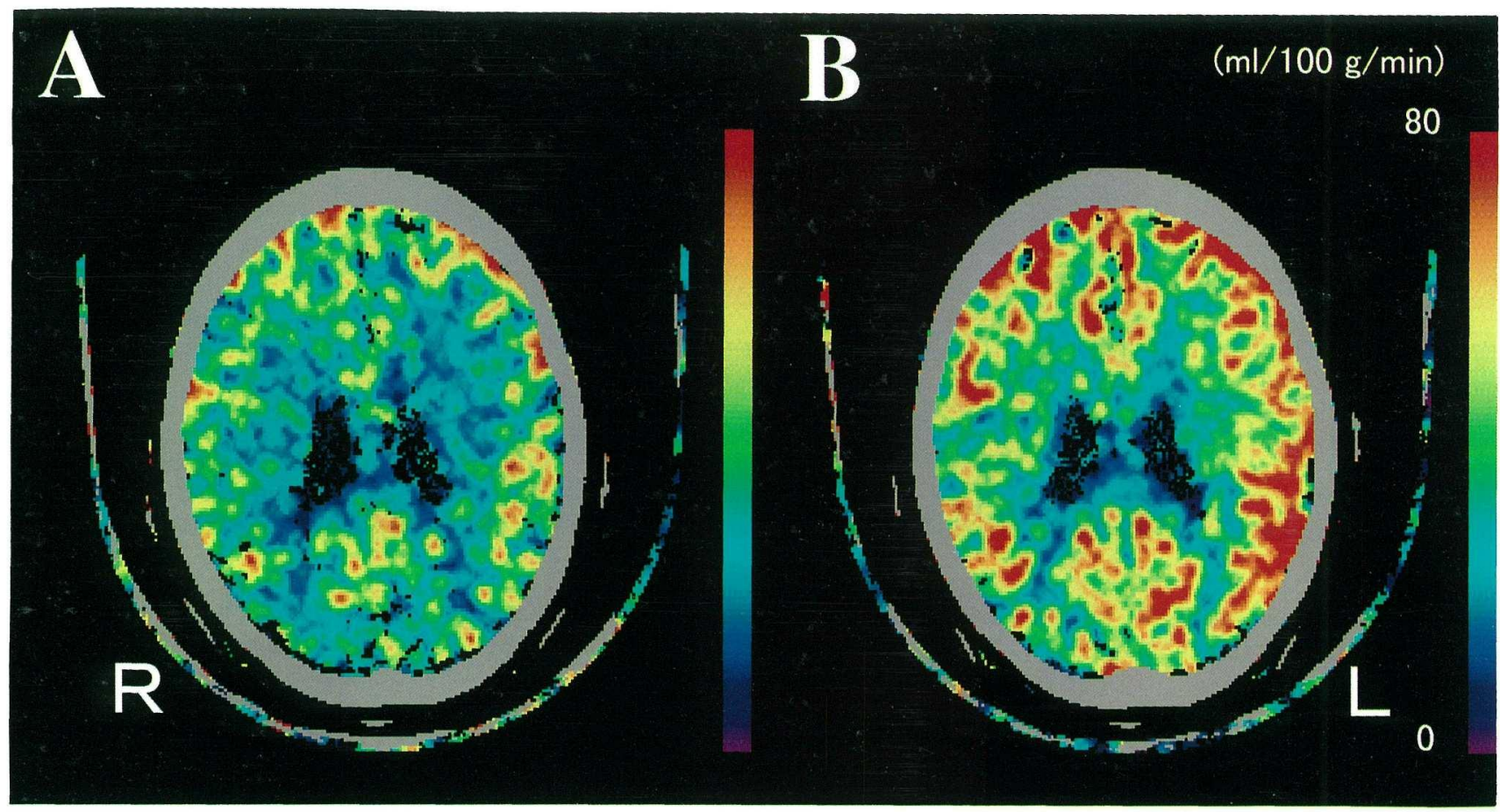

Figure 4. Xenon-enhanced CT visualized hypoperfusion in the bilateral, but more dominantly right parieto-occipital watershed regions in the steady state (A). After intravenous administration of acetazolamide, a vasodilator inhibiting carbonic anhydrase, the reduction in regional cerebral blood flow was more prominently demonstrated (B).

Table 2. Hepatic Encephalopathy Presenting with Focal Higher Cortical Signs

\begin{tabular}{lccll}
\hline \multicolumn{1}{c}{ Author } & Year & Age/Sex & Etiology & \multicolumn{1}{c}{ Focal signs and symptoms } \\
\hline Oda M (18) & 1964 & $56 / \mathrm{F}$ & PSE & Apraxia, Agnosia, Agraphia \\
Fung WP et al (19) & 1968 & $55 / \mathrm{M}$ & Liver cirrhosis & Constructional apraxia \\
& & $48 / \mathrm{M}$ & Liver cirrhosis & Constructional apraxia \\
Kamei et al (20) & 1976 & $60 / \mathrm{M}$ & Liver cirrhosis & Constructional apraxia, Dyscalculia \\
Oyama et al (15) & 1980 & $57 / \mathrm{M}$ & Liver cirrhosis & Dressing and constructional apraxia, Gerstmann syndrome, Visual spatial agnosia \\
& & $48 / \mathrm{F}$ & Liver cirrhosis & Constructional apraxia \\
Kobayashi, et al (17) & 1988 & $54 / \mathrm{M}$ & PSE & Gait disturbance, Dysarthria, Myoclonus, Dressing and constructional apraxia \\
Kita et al (21) & 1989 & 69/M & Liver cirrhosis & Constructional apraxia, Left himiparesis, Finger-agnosia, Dyscalculia \\
Abts et al (22) & 1993 & $52 / ?$ & Liver cirrhosis? & Apraxia, Dyscalculia, Agnosia, Aphasia \\
Present case & 1998 & 72/F & PSE & Dressing and constructional apraxia, Dyscalculia, Left hemiparesis \\
\hline
\end{tabular}

PSE: Portal systemic encephalopathy.

characteristic pathological changes of acquired hepatocerebral disorders, such as spongy degeneration and proliferation of Alzheimer type 2 astrocytes, were more extensive in the boundary arterial territories. In the present case, LCBF was attenuated in bilateral, especially right-sided, parietal watershed regions. Although we cannot tell whether primary alteration of local cerebral circulation or the secondary effect due to the pathological changes is involved in the decrease of LCBF, it may be related to the pathogenesis of the dressing and constructional apraxia observed in this patient. The subtle weakness of the left hand might be explained by a similar mecha- nism in the right corona radiata or precentral cortex in the boundary territory.

Because the patient had a dissecting aneurysm of the descending aorta, conventional angiography had some risk. We, therefore, tried abdominal MR angiography, which successfully detected an intrahepatic portal systemic shunt. Aging is thought to be an exacerbating factor of hepatic encephalopathy presumably due to decreased tolerance of the detrimental metabolites or to lower cerebral perfusion. It is possible that patients with a portal systemic shunt do not present any typical symptoms until they are elderly (24). They could be misdiag- 


\section{Portosystemic Encephalopathy and Apraxia}

nosed as having neurodegenerative or cerebrovascular diseases, as in this case, because they have no history of liver disease. Combined examinations of brain MRI and abdominal MR angiography, in addition to neuropsychological tests, especially for constructional apraxia, may provide a strong tool to detect underlying portal systemic encephalopathy.

Acknowledgements: The authors are indebted to Dr. Shigeru Watanabe, Dr. Keiji Yamaguchi, Dr. Tomohisa Dembo, and Ms. Mayumi Tsukahara for their measurement of LCBF.

\section{References}

1) Rothstein JD, Herlong HF. Neurologic manifestations of hepatic disease. Neurol Clin 7: 563-578, 1989.

2) Podolsky DK, Isselbacher KJ. Major Compilcations of Cirrhosis. in: Harrison's Principles of Internal Medicine. 14th ed. Fauci AS, Braunwald E, Isselbacher KJ, et al, Eds. McGraw-Hill, New York, 1998: 1710-1717.

3) Watanabe A. Cerebral changes in hepatic encephalopathy. J Gastroenterol Hepatol 13: 752-760, 1998.

4) Sen S. Unusual neuropsychiatric manifestations in liver failure. J Indian Med Assoc 51: 434-437, 1968.

5) Atchison JW, Pellegrino M, Herbers P, Tipton B, Matkovic V. Hepatic encephalopathy mimicking stroke. Am J Phys Med Rehabil 71: 114-118, 1992.

6) Imamura J, Ikeyama Y, Kim S, Nagaoka S, Kajii N. A case of portal systemic encephalopathy beginning with left hemiparesis. Iryo 51: 540-543, 1997 (In Japanese, Abstract in English).

7) Hasegawa $K$, Inoue $K$, Moriya $K$. An investigation of dementia rating scale for the elderly. Seishinigaku (Clin Psychiatry) 16: 965-969, 1974 (in Japanese).

8) Inoue $\mathrm{E}$, Hori $\mathrm{S}$, Narumi $\mathrm{Y}$, et al. Portal-systemic encephalopathy: Presence of basal ganglia lesions with high signal intensity on MR images. Radiology 179: 551-555, 1991.

9) Zeneroli ML, Cioni G, Crisi G, Vezzelli C, Ventura E. Globus pallidus alterations and brain atrophy in liver cirrhosis patients with encephalopathy: An MR imaging study. Magn Reson Imaging 9: 295-302, 1991.

10) Brunberg JA, Kanal E, Hirsch W, Van Thiel DH. Chronic acquired hepatic failure: MR imaging of the brain at $1.5 \mathrm{~T}$. AJNR Am J Neuroradiol
12: 909-914, 1991.

11) Adams RD. Acquired hepatocerebral degeneration. in: Handbook of Clinical Neurology. Vol. 6 Diseases of the Basal Ganglia. Vinken PJ, Bruyn GW, Eds. North-Holland Publishing Company, Amsterdam, 1968: 279_ 297.

12) Caplan LR, Scheiner D. Dysconjugate gaze in hepatic coma. Ann Neurol 8: $328-329,1980$.

13) Plum F, Possner JB. Diagnosis of Stupor and Coma. 3rd ed. F. A. Davis Company, Philadelphia, 1980: 222-225.

14) Lishman WA. Organic Psychiatry. The Psychological Consequences of Cerebral Disorder. 2nd ed. Blackwell Scientific Publications, Oxford, 1987: 479-482.

15) Oyama S, Takehara S, Kabashima K, Matsunaga T, Kiyota K. Neuropsychological symptoms in the acquired hepatocerebral degeneration. Seishinigaku (Clin Psychiatry) 22: 607-615, 1980 (in Japanese).

16) Gitlin N. Subclinical portal-systemic encephalopathy. Am J Gastroenterol 83: 8-11, 1988.

17) Kobayashi M, Mizobuchi M, Hirota S, Shoda T. The clinical course of hepato-cerebral degeneration due to portal systemic shunt, as examined by constructional ability of plane and cube. Rinsho Shinkeigaku (Clin Neurol) 28: 125-128, 1988 (in Japanese, Abstract in English).

18) Oda M. A contribution to the clinical and histopathological problem on the hepatocerebral diseases with special reference to "pseudo-uregyria." Seishin Shinkeigaku Zasshi 66: 892-931, 1964 (in Japanese).

19) Fung WP, Khoo OT. Treatment of chronic hepatic encephalopathy with lacturose. Med J Aust 2: 160-163, 1968.

20) Kamei H, Nishimaru Y, Okumura J. A case of recurrent hepatic encephalopathy secondary to gastorectomy. Rinsho To Kenkyu 53: 3677-3683, 1976 (in Japanese).

21) Kita Y, Matsuura T, Ogawa M, Matsubayashi K, Mimori T. A case of hepatic encephalopathy beginning with hemiparesis. Rinsho Shinkeigaku (Clin Neurol) 28: 254, 1988 (in Japanese).

22) Abts H, Crols R, Marien P, Saerens J, Holvoet J, de Deyn P. Paroxystic neuropsychological symptoms as the early expression of hepatic encephalopathy. A case report. Acta Neurol (Napoli) 15: 268-276, 1993 (published erratum appears in Acta Neurol (Napoli) 15: preceding 321, 1993).

23) Finlayson MH, Superville B. Distribution of cerebral lesions in acquired hepatocerebral degeneration. Brain 104: 79-95, 1981.

24) Kerlan RK Jr, Sollenberger RD, Palubinskas AJ, Raskin NH, Callen PW, Ehrenfeld WK. Portal-systemic encephalopathy due to a congenital portocaval shunt. AJR Am J Roentogenol 139: 1013-1015,1982. 